\title{
Morphology, Anatomy, and Development of Cunninghamia lanceolata (Cupressaceae) Pollen Cones.
}

\author{
Brian A. Atkinson ${ }^{1}$, Teresa Sawyer ${ }^{2}$, Gar W. Rothwell ${ }^{1,3}$, and Ruth A, Stockey ${ }^{1}$ \\ 1. Department of Botany and Plant Pathology, Oregon State University, Corvallis, Oregon, USA. \\ 2. Electron Microscope Facility, Oregon State University, Corvallis, Oregon, USA. \\ 3. Department of Environmental and Plant Biology, Ohio University, Athens, Ohio, USA.
}

Cupressaceae (Cypress family) is the most widespread and one of the most economically important conifer lineages [1]. Some well-known representatives of Cupressaceae are Sequoia (redwoods), Juniperus (junipers), and False Cedars (Thuja, Calocedrus, Chamaecyparis). This family has a long and rich fossil history that dates back to the Early Jurassic [2]. Due to its impressively long and complex evolutionary history, Cupressaceae has been a subject of numerous morphological, anatomical, paleobotanical, and evolutionary studies.

The majority of past studies have focused on seed cones within Cupressaceae [3]. In contrast, pollen cone studies are largely lacking, thus our understanding of pollen cone evolution within Cupressaceae is hindered. There is a growing number of paleobotanical studies from several geological time intervals [2, 4] that are beginning to shed light on the evolution of pollen cones. Recently, the paleobotany lab at Oregon State University has recovered cupressaceous pollen cone fossils from the Eocene (ca $55 \mathrm{Ma}$ ) of Western Canada. Coupled with detailed structural and developmental studies of living cones these fossils will provide a greater understanding of the evolution of Cupressaceae and more fully characterize changes in conifer pollen cones through time.

Preliminary results reveal that the fossil pollen cones are assignable to the extant genus, Cunninghamia. This genus represents the earliest diverging lineage within Cupressaceae with an impressive fossil record of seed cones, wood, and leaves. To further our understanding of the evolution of this genus and of the Cupressaceae as a whole, studies of living pollen cones also have been initiated. The immediate goals of this study are as follows: 1) to characterize the morphology and anatomy of young pollen cones in bud stage, 2) to characterize the morphology and anatomy of mature pollen cones, and 3) to reveal any significant changes in cone structure through the entire range of development. The over-arching goal of this research is to provide a structural framework for future studies regarding the morphology, anatomy, and evolution of cupressaceous pollen cones.

Two trees of Cunninghamia lanceolata that are cultivated in Corvallis, Oregon and at Ohio University, Athens, Ohio provided material for study. Two major stages of development are of interest: the late-bud stage and mature pollen cone stage. During January 2015, buds of pollen cone clusters were collected and sectioned transversely and longitudinally before chemical fixation. Sectioned buds were fixed in Formalin - Acetic Acid - Alcohol (FAA) for twelve days. Afterwards, the sectioned pollen cones were dehydrated in an ethanol series (30\% for two days, 50\% for three days, $70 \%$ for four days, and $95 \%$ for four days). After dehydration the specimens were infiltrated and embedded with ethanol-glycol methacrylate (Technovit 7100 embedding kit, Electron Microscopy Sciences, Hatfield, Pennsylvania, USA) resin, at sequential ratios: 2:1, 1:1, and 2:1. Then specimens were fully embedded in the plastic. The embedded specimens were sectioned using an AO 820 rotary microtome at a thickness of 5 microns. Microtome sections were stained with toluidine blue (pH 4.2) and mounted microscope slides 
with mounting medium. Prepared specimens will be photographed using a BetterLight digital scanning camera (Better Light, Placerville, California, USA).

Pollen cone clusters in bud stage will also be dissected, sectioned, and imaged using light microscopy and Scanning Electron Microscopy. Later during the growing season in spring, mature cones will be collected and dissected and studied using SEM, (Quanta 600 FEG (FEI, Hillsboro, OR, USA).

Samples for SEM will be chemically fixed in a modified Karnovsky solution for 60 hours at $4^{0} \mathrm{C}$. Pollen cones will be dehydrated as above in preparation for critical point drying. Samples will mounted to a stub with carbon tape, sputter coated and imaged.

The results from this study will provide important data regarding the morphology, anatomy, and development of pollen cones of Cunninghamia. Due to the phylogenetic position and outstanding fossil record of Cunninghamia, we predict that differences between the young and mature stages of pollen cones will meaningfully improve our understanding of conifer reproductive evolution. Furthermore, the data yielded from this study will provide a framework for working with fossil pollen cones that represent different stages of development. Using a multidisciplinary approach consisting of structural and paleobotanical research we have an opportunity to shed light on the evolution of cupressaceous pollen cones [5].

Images will include early pollen cone development, cross sections of pollen cones, pollen with light and electron microscopes.

\section{References:}

[1] A Farjon and D Filer in "An Atlas of the World's Conifers: An Analysis of Their Distribution, Biogeography, Diversity and Conservation Status" Brill (2013) p. 17.

[2] I Escapa et al, Review of Palabotany and Palynology 151 (2008), p. 110.

[3] BA Atkinson et al, American Journal of Botany 101 (2014), p. 2136.

[4] GR Hernandez-Castillo et al, International Journal of Plant Sciences 166 (2005), p. 339.

[5] The authors acknowledge funding from the National Science Foundation, grant number DGE1314109. 